\title{
Autorregulación del Aprendizaje en Entornos Personales de Aprendizaje en el Grado de Educación Primaria de la Universidad de Granada, España
}

\author{
Eduardo Chaves, Juan M. Trujillo y Juan A. López \\ Universidad de Granada, Facultad de Ciencias de la Educación, Campus de Cartuja s/n - CP 18071, \\ Granada-España (e-mail: eduardochb@ugr.es; jttorres@ugr.es; juanlope@ugr.es) \\ * Autor a quien debe ser dirigida la correspondencia.
}

Recibido Dic. 9, 2014; Aceptado Ene. 20, 2015; Versión final recibida Mar. 9, 2015

\begin{abstract}
Resumen
El objetivo del estudio es analizar el proceso de autorregulación del aprendizaje en entornos personales de aprendizaje. El análisis se enfoca en acciones realizadas y logros obtenidos por los estudiantes durante las fases de actuación y de reflexión de este proceso. Se aplicó un cuestionario, tipo escala Likert, a una muestra aleatoria por conglomerados del grado en Educación Primaria de la Universidad de Granada en España. Sobre los datos se realizan análisis descriptivos e inferenciales, también pruebas no paramétricas de correlaciones y de varianzas. Los resultados muestran que las herramientas digitales y los profesores son elementos importantes en el proceso de autorregulación del aprendizaje. En general, la población es exitosa en ambas fases de este proceso, también hay subgrupos de estudiantes que sobresalen por sus logros. Se concluye que el uso adecuado de las herramientas digitales del entorno personal de aprendizaje favorece la autorregulación del aprendizaje en ambas fases.
\end{abstract}

Palabras clave: autorregulación del aprendizaje, entornos personales de aprendizaje, herramientas digitales

\section{Self-regulated Learning in Personal Learning Environments on the Grade of Elementary Education, University of Granada, Spain}

\begin{abstract}
The objective of the study is to analyze the process of self-regulation of the learning in personal learning environments. The analysis focuses on actions taken and achievements made of students during the phases of action and self-reflection of this process. A Likert scale type questionnaire was applied to a random cluster sample from the degree in Elementary Education of the University of Granada in Spain. The study performed descriptive and inferential analysis of the data, non-parametric tests of correlations and variances. The results show that digital tools and the teachers are important elements in the process of selfregulated learning. In general, the population is successful in both phases of this process. There are also subgroups of students who stand out for their achievements. It is concluded that proper use of digital tools of personal learning environment favors the self-regulation of the learning process in both phases.
\end{abstract}

Keywords: self-regulated learning, personal learning environment, digital tools 


\section{INTRODUCCIÓN}

En la década de los 70's del siglo pasado las teorías educativas centraron su atención en el aprendizaje, en vez de la enseñanza. En la década de los 80 's los pedagogos y los psicólogos comienzan a utilizar el término aprendizaje autorregulado. La aproximación al concepto se ha hecho desde varias perspectivas teóricas, entre ellas la conductista, la socio-constructivista y la socio-cognitiva. Actualmente, en concordancia con esta riqueza conceptual, existen potentes corrientes de investigación relativas al aprendizaje autorregulado que han madurado con el tiempo (Hadwin et al. 2010; Jin and Low, 2009; Pintrich et al., 1993; Zimmerman, 2008).

En el socio-cognitivismo el aprendizaje autorregulado se concibe como un fenómeno donde los individuos sistemáticamente activan y sostienen un proceso cognitivo, motivacional/afectivo y conductual para lograr conocimientos, habilidades y destrezas, en un determinado contexto. Desde esta perspectiva, el individuo que aprende se percibe dentro de un ambiente cambiante que lo obliga a realizar constantes esfuerzos metacognitivos de evaluación y realimentación para ajustar sus conductas y estrategias; consecuentemente, el aprendizaje es visto como una interacción entre elementos intrapersonales, conductuales y ambientales (Zimmerman, 2001; Zimmerman, 1990; Schunk y Zimmerman, 1994; Azevedo, 2007). En este modelo teórico el aprendizaje autorregulado consiste en un proceso cíclico de tres fases llamadas previsión, actuación y reflexión (Zimmerman y Schunk, 1989; Zimmerman, 2000; Schunk y Zimmerman, 2003; Pajares, 2002; Pintrich, 2004; Zimmerman y Cleary, 2006; Usher y Pajares, 2008; Zimmerman y Cleary, 2009).

En la fase de previsión el individuo selecciona y ajusta los objetivos de aprendizaje, también realiza una planificación estratégica. En la fase de actuación, también llamada de control volitivo, se materializan las estrategias de la primera fase y el sujeto lleva a cabo sus tareas para cumplir con sus objetivos de aprendizaje. Paralelamente, esta fase involucra actuaciones volitivas de autocontrol y de autobservación, porque el sujeto realiza acciones intencionales de inspección y observación sobre el rendimiento y la eficacia de sus conductas, sobre las estrategias que está aplicando y sobre el logro que está alcanzando en sus objetivos de aprendizaje. En la última fase, la de reflexión, el individuo realiza procesos de autoevaluación y de autojuicio, es decir, utiliza las observaciones y las experiencias obtenidas en la fase de actuación para tomar decisiones sobre sus objetivos, sus estrategias y sus acciones, realimentando los nuevos ciclos de autorregulación del aprendizaje.

El enorme auge de las herramientas digitales ha despertado el interés de los teóricos y de los investigadores por estudiar el aporte de estas tecnologías en el aprendizaje, véanse como ejemplos a Lechuga et al. (2014), Fredes et al. (2012) y Tovar et al. (2014). Por su parte, las teorías relacionadas con el aprendizaje autorregulado gozan de una renovada vigencia en el ámbito de la tecnología aplicada a la educación; un claro ejemplo de esta tendencia es la idea de entorno personal de aprendizaje (PLE, Personal Learning Environments).

Se conoce como PLE al conjunto de herramientas, físicas y virtuales, que una persona utiliza en la vida cotidiana para aprender (Attwell, 2007b). Detrás de la investigación y la teorización sobre el PLE subyace un enfoque pedagógico para la utilización de las herramientas digitales que reconoce un papel activo y proactivo en la persona y que pone especial atención en la reflexión, la metacognición, y muy especialmente a la autorregulación del aprendizaje en diversos contextos.

La teoría sobre PLE sostiene que el individuo, en su entorno personal, puede ejercitar la capacidad de autorregular su propio aprendizaje; por ejemplo, fijar sus propias metas, elegir y organizar sus propios contenidos, comunicarse con otros estudiantes y con profesores durante sus gestiones y, finalmente, lograr el cumplimiento de sus objetivos de aprendizaje (Väljataga y Laanpere, 2010; Chatti et al., 2010; Attwell, 2007a; Attwell, 2007b, Wilson, 2008; Drexler, 2010; Wilson et al., 2007; Johnson y Sherlock, 2014). Es decir, en su PLE el individuo puede llevar a cabo las tres fases del aprendizaje autorregulado; el PLE es el ambiente donde el sujeto puede desplegar su iniciativa, su tenacidad y su adaptabilidad para desarrollar la autorregulación de su aprendizaje.

\section{EL ESTUDIO REALIZADO}

El objetivo general del trabajo es analizar las fases de actuación y de reflexión del proceso de autorregulación del aprendizaje en PLE, enfocándose en las acciones realizadas y los logros alcanzados por una población. La población está conformada por los estudiantes del Grado de Maestro en Educación Primaria (GEP) de la Universidad de Granada (UGR), de España.

Para cumplir el objetivo general se plantean cinco objetivos específicos. El primero es determinar acciones que realizan los estudiantes del GEP para autorregular el aprendizaje en sus PLE, durante las fases de 
actuación y reflexión. El segundo objetivo es establecer las correlaciones que existen entre estas acciones. El tercer objetivo es identificar logros que han alcanzado los estudiantes del GEP durante las fases de actuación y reflexión, del proceso de autorregulación del aprendizaje en PLE. El cuarto objetivo es correlacionar estos logros. El último objetivo es analizar las influencias que tienen las acciones, determinadas en el primer objetivo, sobre los logros, identificadas en el tercer objetivo.

Los resultados del estudio son importantes para los estudiantes porque pueden ayudarlos en el conocimiento y desarrollo de sus propios PLE. La teoría sobre el aprendizaje autorregulado reconoce que la percepción del sujeto sobre su propia eficiencia para alcanzar el éxito en el aprendizaje es muy importante, porque orienta o motiva al individuo a continuar con el ciclo del aprendizaje autorregulado (Zimmerman y Cleary, 2006; Pajares, 2007; Usher y Pajares, 2008; Zimmerman, 2008; Jin y Low, 2009; Bandura, 1997). Estudios empíricos de este tipo pueden ayudar a los estudiantes en la percepción de su propia eficiencia en el aprendizaje, pues ésta implica una conciencia metacognitiva del sujeto sobre las actividades de aprendizaje, las propias conductas, los logros cognitivos alcanzados y las estrategias elegidas y empleadas (Schunk y Zimmerman, 2003).

Las cuestiones pedagógicas que se exponen en este trabajo son de interés para los académicos encargados del GEP, y en general, para educadores, investigadores, administradores de la educación y encargados de cualquier programa de formación docente. El modelo socio-cognitivo del aprendizaje autorregulado reconoce la importancia de profesores, de investigadores, de expertos y de las redes sociales en el proceso de autorregulación de las conductas de los estudiantes (Zimmerman, 2008).

Los teóricos e investigadores sobre PLE consideran muy importante investigar, evaluar y hacer visible todo proceso que se lleve a cabo en el PLE, porque se considera que los profesores, los encargados de programas de formación, los compañeros condiscípulos, las comunidades educativas, las redes sociales y la inteligencia colectiva de internet brindan un apoyo decisivo en el uso consciente, reflexivo y autorregulado de las herramientas para alcanzar los aprendizajes. Los profesores, los expertos y las redes sociales son fundamentales para lograr que el PLE, de cada individuo, sea cada vez más eficiente, personalizado y satisfactorio (Ebner y Taraghi, 2010; Godwin-Jones, 2009; Dabbagh y Kitsantas, 2012; Johnson y Liber, 2008; Liber y Johnson, 2008; Archee, 2012); en este sentido, es posible hablar de una responsabilidad global.

\section{METODOLOGÍA}

\section{Población y muestra}

La población de estudiantes activos en el GEP de la UGR tiene un total de 1774 sujetos, entre estos 1004 son mujeres y 770 hombres. La población está dividida en 28 grupos de estudiantes de tamaño homogéneo (alrededor de 63 estudiantes), 9 grupos son del primer curso, 10 del segundo y 9 del tercero. Estos grupos de estudiantes han servido como marco de conglomerados para seleccionar aleatoriamente la muestra del estudio, que está compuesta por 15 grupos con 668 estudiantes. Esta muestra permite el cálculo de los intervalos de confianza (IC) al 95\%. Sobre esta muestra de estudiantes se ha aplicado un cuestionario al finalizar el ciclo lectivo, durante los meses de mayo y junio de 2013.

\section{El cuestionario}

El cuestionario se elaboró con base a las teorías de autorregulación del aprendizaje y del PLE. Tiene preguntas sobre aspectos generales, estas son sexo, edad, cantidad de horas semanales dedicadas a diferentes actividades y tenencia de dispositivos para el uso personal (ordenador portátil, tableta y teléfono inteligente, entre otros). Las variables sobre aspectos generales se utilizan para caracterizar la población.

Los siguientes veintisiete ítems del cuestionario preguntan a los estudiantes sobre las acciones realizadas (16 ítems) y los logros alcanzados (11 ítems) durante las fases de actuación y de reflexión del proceso de autorregulación del aprendizaje. Estos ítems se denominan ítems principales, son tipo escala Likert, con cuatro opciones (muy en desacuerdo, en desacuerdo, de acuerdo y muy de acuerdo).

\section{Variables, pruebas e hipótesis}

A las preguntas generales del cuestionario le corresponden variables dicotómicas y de razón, a los ítems principales les corresponden veintisiete variables ordinales de cuatro niveles. A estas últimas se les denomina variables principales porque son las que se analizan para el cumplimiento de los objetivos. 
Para lograr el cumplimiento de los dos primeros objetivos del estudio se ha realizado estadística descriptiva e inferencial con las variables principales del cuestionario. Para el cumplimiento de los siguientes tres objetivos se aplican e interpretan pruebas no paramétricas para el análisis de correlaciones y de varianzas de las variables principales, respectivamente el coeficiente de correlación de Kendall (Leech, Barrett, y Morgan, 2011; Howell, 2010) y la prueba Kruskal-Wallis (Kruskal y Wallis, 1952), ambas pruebas al 95\% con significación bilateral. Para las pruebas de correlación se plantean las hipótesis de correlación lineal para cada par de acciones, y los mismo se hace para cada par de logros. En estos casos cada hipótesis nula dice que el índice de correlación es cero, cada hipótesis alternativa dice que el índice es diferente de cero. El resultado es una matriz de correlaciones, se incluyen en el artículo solo las correlaciones estadísticamente significativas.

La prueba de análisis de varianza Kruskal-Wallis se aplica para establecer la influencia que tienen las acciones para autorregular los aprendizajes en PLE sobre cada uno de los logros alcanzados en este proceso. Es decir, para cada par acción-logro se plantea como hipótesis nula la igualdad de todas las medidas en la variable logro en los distintos niveles del factor acción, y como hipótesis alternativa la no igualdad de todas la medidas. En estos casos la variable independiente es la acción y la variable dependiente es el logro. En cada prueba de análisis de varianza se ha calculado y analizado el tamaño del efecto ES (Cohen, 1992; Strahan, 1982) con un nivel de confianza del 95\%.

\section{Validez y confiabilidad del cuestionario}

El cuestionario tiene la validación de diez expertos en las temáticas que plantea, la información que ofrecieron los expertos fue analizada cuantitativa y cualitativamente, en el primer caso utilizando el índice de congruencia de Osterlind, en el segundo caso mediante procesos de codificación y categorización, lo que permitió que se incorporaran sugerencias de los expertos al cuestionario. Todos los ítems del cuestionario tienen un índice de congruencia mayor a 0.5 , esto indica que el instrumento es válido. Posteriormente, se realizó una aplicación piloto del cuestionario. La aplicación piloto del cuestionario permitió analizar la confiabilidad del instrumento, mediante el coeficiente alfa de Cronbach. El valor del alfa del instrumento es de 0.927 , su confiabilidad es excelente.

\section{RESULTADOS}

\section{Acciones para la autorregulación del aprendizaje en PLE}

La muestra de estudiantes del GEP utilizada en el estudio está constituida por un $61.6 \%$ de mujeres y un $38.4 \%$ de hombres, con un error muestral comprobado del $5 \%$. La edad promedio es de $21.89 \pm 0.79$ años cumplidos, un $94.91 \% \pm 1.8$ tiene ordenador portátil, $86.37 \% \pm 2.70$ tiene teléfono inteligente, un $48.89 \% \pm$ 3.78 tiene ordenador de sobremesa. En promedio dedican semanalmente $19.39 \pm 3.86$ horas a utilizar las herramientas que conforman sus PLE y $6,57 \pm 1.46$ horas a visitar alguna plataforma en red de la universidad.

La Tabla 1 muestra las acciones para la autorregulación del aprendizaje que han realizado los estudiantes en sus PLE durante la fase de actuación, por lo tanto, incluye las acciones que realizan los estudiantes para cumplir con sus objetivos de aprendizaje y para inspeccionar la eficacia de sus conductas. Los datos en cursiva de la Tabla 1 muestran que hay proporciones significativas de estudiantes que no adaptan las herramientas LMS (Learning Management System, plataformas para la gestión del aprendizaje) a sus metas de aprendizaje (35.93\%) o que no incorporan a su PLE herramientas de estas plataformas $(25.75 \%)$.

En la Figura 1 y la Figura 2 se resumen algunos resultados de la Tabla 1. Se observa que los estudiantes utilizan herramientas externas a la universidad y herramientas LMS para intercambiar información con las personas participantes en la asignatura, siendo las primeras preferidas sobre las segundas. El uso de las herramientas externas sobresalen por la cantidad (90.27\%, estudiantes "De acuerdo" y "Muy de acuerdo") y por la intensidad (51.65\% estudiantes "Muy de acuerdo"). También se evidencia que, en porcentajes significativos, los estudiantes intercambian información con personas externas a la asignatura utilizando sus herramientas PLE.

Como se muestra en la Figura 2, en la fase de actuación los estudiantes documentaron sus procesos personales de aprendizajes, una acción volitiva de autocontrol y de autobservación que permite recabar datos que sustentarán, posteriormente, las acciones durante la fase de reflexión. En la fase de actuación las herramientas digitales del PLE son muy importantes para representar las ideas de formas diversas. Igualmente, el profesor de la asignatura forma una parte importante del entorno de aprendizaje de los estudiantes, los estudiantes acuden a él para recibir realimentación y llevar a cabo acciones de autocontrol. Cabe resaltar que en esta labor los estudiantes del GEP muestran un pensamiento crítico ante las estrategias de enseñanza de los docentes. 
Tabla 1: Acciones para la autorregulación del aprendizaje en PLE, fase de actuación

En la Tabla, $\mathrm{N}=1774$, (1) IC 95\% y (2) $N R=$ No respuesta

\begin{tabular}{|c|c|c|c|c|c|}
\hline \multirow[b]{2}{*}{ Acciones } & \multicolumn{4}{|c|}{ Frecuencias relativas con intervalos de confianza ${ }^{(1)}$} & \multirow{2}{*}{$\begin{array}{c}\% \\
N R^{(2)}\end{array}$} \\
\hline & $\begin{array}{c}\text { Muy en } \\
\text { desacuerdo }\end{array}$ & $\begin{array}{c}\text { En } \\
\text { desacuerdo }\end{array}$ & $\begin{array}{l}\mathrm{De} \\
\text { acuerdo }\end{array}$ & $\begin{array}{l}\text { Muy de } \\
\text { acuerdo }\end{array}$ & \\
\hline $\begin{array}{l}\text { tilizar herramientas externas a la universidad para } \\
\text { tercambiar información con personas participantes } \\
\text { n la asignatura }\end{array}$ & $\begin{array}{c}1.35 \\
{[0.49,2.21]}\end{array}$ & $\begin{array}{c}5.39 \\
{[3.70,7.07]}\end{array}$ & $\begin{array}{c}38.62 \\
{[35.02,42.22]}\end{array}$ & $\begin{array}{c}51.65 \\
{[47.98,55.32]}\end{array}$ & 2.99 \\
\hline $\begin{array}{l}\text { Jtilizar herramientas de alguna } \\
\text { Universidad para dar informacic } \\
\text { barticipantes en la asignatura }\end{array}$ & $\begin{array}{c}1.80 \\
{[0.80,2.79]}\end{array}$ & $\begin{array}{c}9.88 \\
{[7.66,12.11]}\end{array}$ & $\begin{array}{c}51.50 \\
{[47.83,55.17]}\end{array}$ & $\begin{array}{c}33.83 \\
{[30.33,37.34]}\end{array}$ & 2.99 \\
\hline $\begin{array}{l}\text { Jtilizar herramient } \\
\text { deas en formas di }\end{array}$ & $\begin{array}{c}1.65 \\
{[0.70,2.60]}\end{array}$ & $\begin{array}{c}10.93 \\
{[8.60,13.25]}\end{array}$ & $\begin{array}{c}51.65 \\
{[47,98,55,31]}\end{array}$ & $\begin{array}{c}32.63 \\
{[29,16,36.11]}\end{array}$ & 3.14 \\
\hline $\begin{array}{l}\text { Utilizar herramientas de alguna plataforma LMS de la } \\
\text { Universidad para recibir información de personas } \\
\text { participantes en la asignatura }\end{array}$ & $\begin{array}{c}2.40 \\
{[1.25,3.54]}\end{array}$ & $\begin{array}{c}13.32 \\
{[10.79,15.86]}\end{array}$ & $\begin{array}{c}52.40 \\
{[48.73,56.06]}\end{array}$ & $\begin{array}{c}28.89 \\
{[25.53,32.26]}\end{array}$ & 2.99 \\
\hline Solicitar apoyo del profesor de la asignatura & $\begin{array}{c}1.80 \\
{[0.81,2.78]}\end{array}$ & $\begin{array}{c}11.08 \\
{[8.75,13.41]}\end{array}$ & $\begin{array}{c}58.38 \\
{[54.82,61.95]}\end{array}$ & $\begin{array}{c}25.00 \\
{[21.80,28.20]}\end{array}$ & 3.74 \\
\hline $\begin{array}{l}\text { Utilizar herramientas del PLE para } \\
\text { personas externas a la asignatura }\end{array}$ & $\begin{array}{c}4.34 \\
{[2.82,5.86]}\end{array}$ & $\begin{array}{c}22.31 \\
{[19.21,25.40]}\end{array}$ & $\begin{array}{c}45.81 \\
{[42.14,49.48]}\end{array}$ & $\begin{array}{c}24.55 \\
{[21.35,27.75]}\end{array}$ & 2.99 \\
\hline $\begin{array}{l}\text { Utilizar herram } \\
\text { de personas e }\end{array}$ & $\begin{array}{c}4.19 \\
{[2.70,5.69]}\end{array}$ & $\begin{array}{c}22.16 \\
{[19.07,25.24]}\end{array}$ & $\begin{array}{c}48.05 \\
{[44.38,51.73]}\end{array}$ & $\begin{array}{c}22.46 \\
{[19.36,25.55]}\end{array}$ & 3.14 \\
\hline $\begin{array}{l}\text { Mostrar u } \\
\text { enseñanz }\end{array}$ & $\begin{array}{c}1.65 \\
{[0.74,2.55]}\end{array}$ & $\begin{array}{c}12.57 \\
{[10.24,14.91]}\end{array}$ & $\begin{array}{c}53.29 \\
{[50.02,56.56]}\end{array}$ & $\begin{array}{c}20.66 \\
{[17.83,23.49]}\end{array}$ & 11.83 \\
\hline $\begin{array}{l}\text { Incorporar al PL } \\
\text { LMS de la Unive }\end{array}$ & $\begin{array}{c}4.19 \\
{[2.70,5.68]}\end{array}$ & $\begin{array}{c}21.56 \\
{[18.50,24.61]}\end{array}$ & $\begin{array}{c}56.44 \\
{[52.82,60.05]}\end{array}$ & $\begin{array}{c}14.52 \\
{[11.90,17.14]}\end{array}$ & 3.29 \\
\hline ocu & $\begin{array}{c}5.69 \\
{[3.96,7.41]}\end{array}$ & $\begin{array}{c}31.59 \\
{[28.15,35.02]}\end{array}$ & $\begin{array}{c}51.95 \\
{[48.30,55.60]}\end{array}$ & $\begin{array}{c}7.34 \\
{[5.40,9.28]}\end{array}$ & 3.44 \\
\hline $\begin{array}{l}\text { daptar las herramientas de alguna plataforma LMS } \\
\text { e la Universidad a las metas de aprendizaje }\end{array}$ & $\begin{array}{c}6.14 \\
{[4.35,7.93]}\end{array}$ & $\begin{array}{c}29.79 \\
{[26.40,33.18]}\end{array}$ & $\begin{array}{c}54.94 \\
{[51.30,58.58]}\end{array}$ & $\begin{array}{c}5.99 \\
{[4.22,7.76]}\end{array}$ & 3.14 \\
\hline
\end{tabular}

= Muy de acuerdo/De acuerdo = Muy en desacuerdo / En desacuerdo INR

Utilizar herramientas externas a la universidad para intercambiar información con personas participantes en la asignatura

Utilizar herramientas de alguna plataforma LMS de

la Universidad para dar información a personas participantes en la asignatura

Utilizar herramientas de alguna plataforma LMS de

la Universidad para recibir información de personas participantes en la asignatura

Utilizar herramientas del PLE para dar información a personas externas a la asignatura

Utilizar herramientas del PLE para recibir información de personas externas a la asignatura

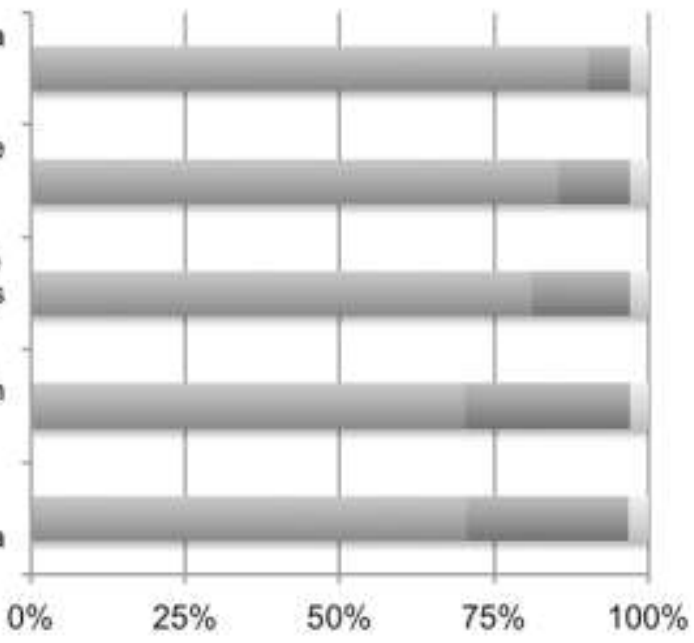

Fig. 1: Acciones para el cumplimiento de objetivos durante la fase actuación 


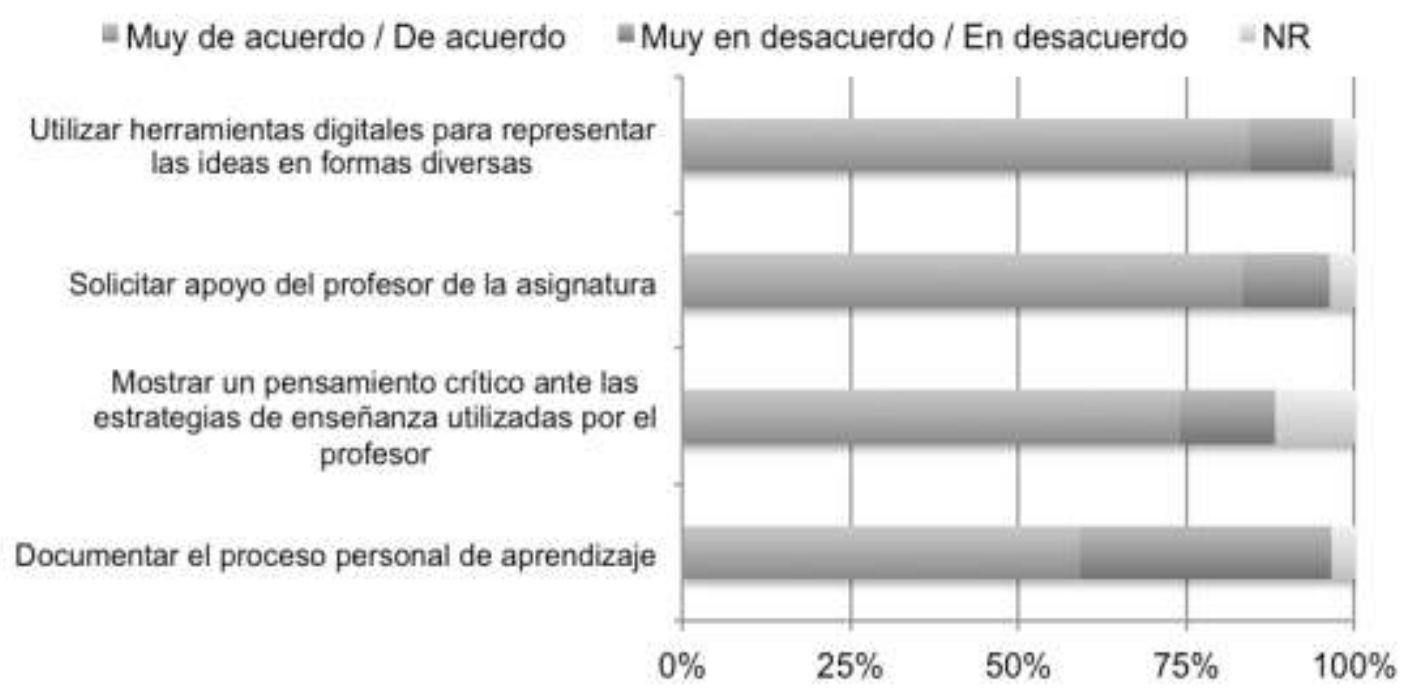

Fig. 2: Acciones para el autocontrol durante la fase de actuación

La Tabla 2 muestra las acciones de los estudiantes durante la fase de reflexión. Según la teoría, los procesos de autoevaluación y de autojuicio que se llevan a cabo en esta fase favorecen que el estudiante aproveche sus experiencias para modificar sus conductas, reformar sus estrategias y para continuar con nuevos ciclos del proceso de autorregulación de sus aprendizajes. Los datos en cursiva de la Tabla 2 señalan que apenas la mitad de los estudiantes del GEP registran las reflexiones sobre su propio aprendizaje $(53.44 \%)$ y solamente algunos $(28.59 \%)$ utilizan un blog para profundizar ideas sobre la asignatura.

Tabla 2: Acciones para la autorregulación del aprendizaje en PLE, fase de reflexión.

En la Tabla, $N=1774$, (1) IC 95\% y (2) NR = No respuesta

\begin{tabular}{lccccc}
\hline & \multicolumn{3}{c}{ Frecuencias relativas con intervalos de confianza } & & \% \\
Acciones & $\begin{array}{c}\text { Muy en } \\
\text { desacuerdo }\end{array}$ & $\begin{array}{c}\text { En } \\
\text { desacuerdo }\end{array}$ & $\begin{array}{c}\text { De } \\
\text { acuerdo }\end{array}$ & $\begin{array}{c}\text { Muy de } \\
\text { acuerdo }\end{array}$ & NR $^{(2)}$ \\
\hline $\begin{array}{l}\text { Valorar las evaluaciones o sugerencias del } \\
\text { profesor para mejorar los trabajos }\end{array}$ & 1.20 & 8.08 & 57.93 & 29.79 & 2.99 \\
& {$[0.39,2.01]$} & {$[6.05,10.12]$} & {$[54.33,61.54]$} & {$[26.40,33.18]$} & \\
$\begin{array}{l}\text { Organizar las reflexiones sobre el aprendizaje } \\
\text { con herramientas digitales }\end{array}$ & 2.84 & 22.90 & 54.49 & 16.77 & 2.99 \\
& {$[1.60,4.09]$} & {$[19.78,26.03]$} & {$[50.84,58.14]$} & {$[13.98,19.55]$} & \\
$\begin{array}{l}\text { Reflexionar sobre el papel que tiene en el } \\
\text { propio aprendizaje }\end{array}$ & 1.65 & 18.26 & 60.93 & 15.87 & 3.29 \\
& {$[0.70,2.60]$} & {$[15.39,21.13]$} & {$[57.39,64.47]$} & {$[13.15,18.58]$} & \\
$\begin{array}{l}\text { Registrar las reflexiones sobre el propio } \\
\text { aprendizaje }\end{array}$ & 4.34 & 39.07 & 45.21 & 8.23 & 3.14 \\
& {$[2.82,5.86]$} & {$[35.47,42.68]$} & {$[41.55,48.87]$} & {$[6.19,10.28]$} & \\
$\begin{array}{l}\text { Utilizar un blog personal para profundizar } \\
\text { ideas o conceptos de la asignatura }\end{array}$ & 22.16 & 45.66 & 23.05 & 5.54 & 3.59 \\
\hline
\end{tabular}

Sin embargo, los estudiantes del GEP realizan la acción de reflexionar sobre el papel que tienen en su propio aprendizaje, como se aprecia en la síntesis que ofrece la Figura 3. En esta figura queda patente que los estudiantes organizan estas reflexiones utilizando herramientas digitales y que valoran las realimentaciones del profesor. Con esto se reafirma la importancia de las herramientas digitales y del profesor en el PLE de los estudiantes y en sus procesos de autorregulación. 


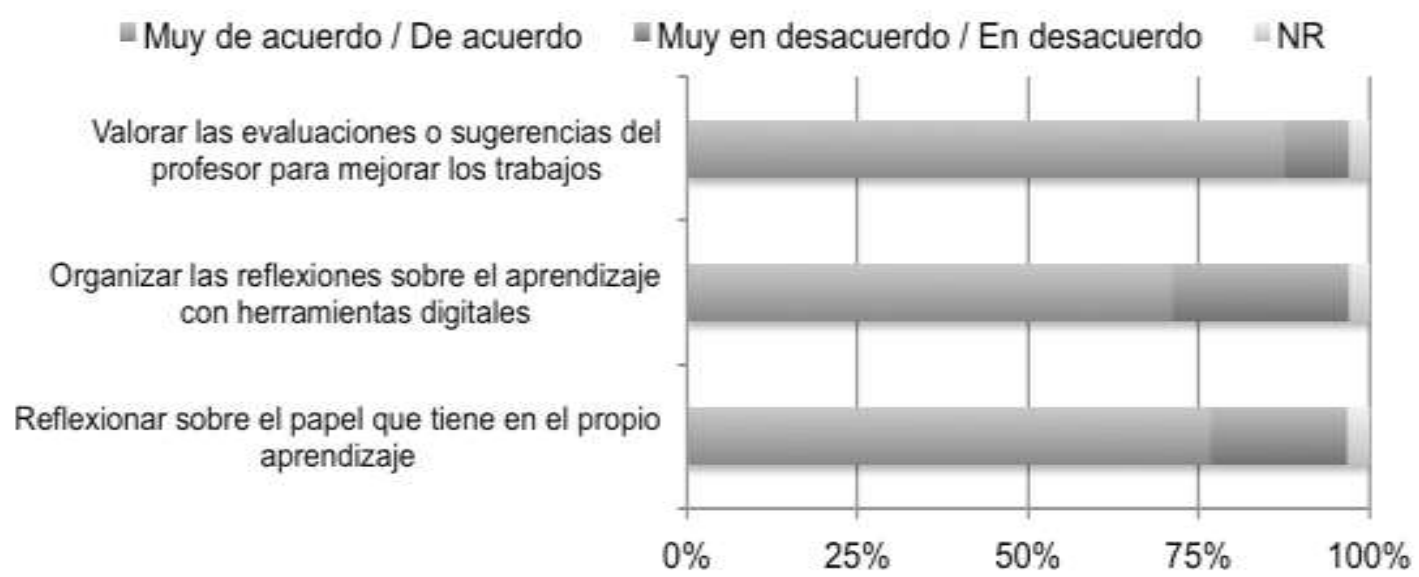

Fig. 3: Acciones para la autorregulación del aprendizaje en PLE, fase de reflexión

\section{Relación entre las acciones de los estudiantes}

La Tabla 3 muestra las correlaciones entre las acciones para la autorregulación del aprendizaje en PLE, los resultados más significativos (mayores o iguales a .30) se muestran en cursiva. De estos datos se desprende que la acción de dar y la acción de recibir información de personas externas a la asignatura tienen una elevada correlación positiva, en ambos casos utilizando las herramientas del PLE.

Tabla 3: Relación entre las acciones para la autorregulación del aprendizaje en PLE.

En la Tabla, $N=1774$, (1) $p<0,01$, dos colas y (2) IC 95\%

\begin{tabular}{|c|c|c|c|c|c|c|c|}
\hline \multirow{2}{*}{\multicolumn{2}{|c|}{ Acciones }} & \multicolumn{6}{|c|}{$\begin{array}{l}\text { Coeficiente de correlación de Kendall(1) e intervalos de } \\
\qquad \text { confianza }^{(2)}\end{array}$} \\
\hline & & 1 & 2 & 3 & 4 & 5 & 6 \\
\hline 1 & $\begin{array}{l}\text { Utilizar herramientas digitales para representar las } \\
\text { ideas en formas diversas }\end{array}$ & & & & & & \\
\hline 2 & $\begin{array}{l}\text { Utilizar herramientas del PLE para dar información a } \\
\text { personas externas a la asignatura }\end{array}$ & $\begin{array}{c}.18 \\
{[.13, .23]}\end{array}$ & & & & & \\
\hline 3 & $\begin{array}{l}\text { Adaptar las herramientas de alguna plataforma LMS } \\
\text { de la Universidad a las metas de aprendizaje }\end{array}$ & $\begin{array}{c}.12 \\
{[.06, .17]}\end{array}$ & $\begin{array}{c}.10 \\
{[.04, .14]}\end{array}$ & & & & \\
\hline 4 & $\begin{array}{l}\text { Valorar las evaluaciones o sugerencias del profesor } \\
\text { para mejorar los trabajos }\end{array}$ & $\begin{array}{c}.38 \\
{[.32, .44]}\end{array}$ & $\begin{array}{c}.20 \\
{[.15, .25]}\end{array}$ & $\begin{array}{c}.09 \\
{[.04, .14]}\end{array}$ & & & \\
\hline 5 & $\begin{array}{l}\text { Utilizar herramientas de alguna plataforma LMS de la } \\
\text { Universidad para recibir información de personas } \\
\text { participantes en la asignatura }\end{array}$ & $\begin{array}{c}.28 \\
{[.22, .33]}\end{array}$ & $\begin{array}{c}.20 \\
{[.15, .25]}\end{array}$ & $\begin{array}{c}.27 \\
{[.22, .32]}\end{array}$ & $\begin{array}{c}.21 \\
{[.16, .26]}\end{array}$ & & \\
\hline 6 & $\begin{array}{l}\text { Utilizar herramientas de alguna plataforma LMS de la } \\
\text { Universidad para dar información a personas } \\
\text { participantes en la asignatura }\end{array}$ & $\begin{array}{l}.31 \\
{[.25, .37]}\end{array}$ & $\begin{array}{l}.14 \\
{[.08, .19]}\end{array}$ & $\begin{array}{c}.20 \\
{[.15, .25]}\end{array}$ & $\begin{array}{l}.31 \\
{[.27, .37]}\end{array}$ & $\begin{array}{c}.57 \\
{[.51, .62]}\end{array}$ & \\
\hline 7 & $\begin{array}{l}\text { Utilizar herramientas externas a la universidad para } \\
\text { intercambiar información con personas participantes } \\
\text { en la asignatura }\end{array}$ & $\begin{array}{c}.28 \\
{[.23, .33]}\end{array}$ & $\begin{array}{c}.34 \\
{[.28, .40]}\end{array}$ & - & $\begin{array}{c}.25 \\
{[.19, .30]}\end{array}$ & $\begin{array}{c}.38 \\
{[.31, .43]}\end{array}$ & $\begin{array}{c}.40 \\
{[.34, .45]}\end{array}$ \\
\hline 8 & $\begin{array}{l}\text { Utilizar herramientas del PLE para recibir información } \\
\text { de personas externas a la asignatura }\end{array}$ & $\begin{array}{c}.19 \\
{[.14, .24]}\end{array}$ & $\begin{array}{c}.66 \\
{[.60, .71]}\end{array}$ & $\begin{array}{c}.10 \\
{[.05, .15]}\end{array}$ & $\begin{array}{c}.19 \\
{[.14, .24]}\end{array}$ & $\begin{array}{c}.20 \\
{[.14, .25]}\end{array}$ & $\begin{array}{c}.09 \\
{[.19, .25]}\end{array}$ \\
\hline 9 & $\begin{array}{l}\text { Incorporar al PLE herramientas de alguna plataforma } \\
\text { LMS de la Universidad }\end{array}$ & $\begin{array}{l}.21 \\
{[.16, .26]}\end{array}$ & $\begin{array}{l}.20 \\
{[.15, .26]}\end{array}$ & {$[.27, .38]$} & $\begin{array}{l}.22 \\
{[.17, .27]}\end{array}$ & $\begin{array}{l}.24 \\
{[.19, .29]}\end{array}$ & $\begin{array}{l}.24 \\
{[.19, .25]}\end{array}$ \\
\hline 10 & Documentar el proceso personal de aprendizaje & $\begin{array}{c}.09 \\
{[.04, .14]}\end{array}$ & $\begin{array}{l}.10 \\
{[.05, .15]}\end{array}$ & $\begin{array}{l}.35 \\
{[.29, .40]}\end{array}$ & $\begin{array}{c}.10 \\
{[.04, .15]}\end{array}$ & $\begin{array}{l}.17 \\
{[.11, .21]}\end{array}$ & $\begin{array}{c}.15 \\
{[.09, .20]}\end{array}$ \\
\hline 11 & $\begin{array}{l}\text { Utilizar un blog personal para profundizar ideas o } \\
\text { conceptos de la asignatura }\end{array}$ & $\begin{array}{l}.07 \\
{[.02, .13]}\end{array}$ & $\begin{array}{c}.09 \\
{[.04, .14]}\end{array}$ & $\begin{array}{c}.38 \\
{[.32, .44]}\end{array}$ & - & $\begin{array}{l}.11 \\
{[.06, .16]}\end{array}$ & $\begin{array}{l}.07 \\
{[.02, .12]}\end{array}$ \\
\hline 12 & $\begin{array}{l}\text { Reflexionar sobre el papel que tiene en el propio } \\
\text { aprendizaje }\end{array}$ & $\begin{array}{c}.42 \\
{[.36, .47]}\end{array}$ & - & - & - & - & - \\
\hline
\end{tabular}


Los datos muestran ternas de acciones relacionadas entre sí. Por ejemplo, la acción de dar y la acción de recibir información de compañeros de asignatura utilizando las herramientas de LMS universitarios tienen una elevada correlación positiva entre sí, y además, ambas tienen correlación con el utilizar herramientas externas a la universidad para intercambiar información con sus compañeros. Esta triple correlación positiva sugiere que, en los procesos de intercambiar información con los compañeros durante la fase de actuación, la utilización de herramientas LMS y la utilización de herramientas externas no son sustitutas sino complementarias.

También hay una correlación significativa entre adaptar herramientas de alguna LMS a las metas de aprendizaje y la acción de incorporar herramientas de la LMS al propio PLE. Esto ilustra una de las razones que tienen los estudiantes para incorporar una herramienta a sus PLE, también puede ayudar a explicar el por qué algunas herramientas LMS son populares y otras no. Son significativas las correlaciones entre la acción de utilizar herramientas digitales para representar las ideas en formas diversas (que es de la fase de actuación) y dos de las acciones que ocurren en la fase de reflexión, cuales son el reflexionar sobre el papel que tienen los estudiantes en su propio aprendizaje y el valorar las evaluaciones y sugerencias del profesor para mejorar el trabajo. Esto indica que el poder ver las ideas de formas diversas durante la fase de actuación se relaciona con los procesos de metacognición durante la última fase del aprendizaje autorregulado.

\section{Logros en la autorregulación del aprendizaje en PLE}

Los datos de la Tabla 4 muestran que los estudiantes del GEP son exitosos en la fase de actuación. La mayoría de los estudiantes han logrado trabajar organizadamente en sus tareas, leer los materiales entregados por el profesor y entregar los trabajos en los plazas señalados. También, desde un punto de vista informático, han logrado utilizar exitosamente las herramientas tecnológicas y la información durante la fase de actuación.

Tabla 4: Logros durante las fases de actuación y reflexión del proceso de autorregulación del aprendizaje en PLE. En la Tabla, $N=1774$, (1) IC 95\% y (2) NR = No respuesta

Frecuencias relativas con intervalos de confianza ${ }^{(1)}$

$\%$

Logros

Muy en

desacuerdo

En

De
acuerdo

Muy de acuerdo

$N R^{(2)}$

Fase de actuación

Cumplió con los plazos para entregar los trabajos

$\begin{array}{lllll}0.45 & 2.54 & 28.29 & 68.11 & 0.60\end{array}$

Logró una adecuada dosificación del tiempo para realizar sus tareas

Realizó a tiempo las actividades propuestas por el profesor para trabajo extra clase

Utilizó exitosamente herramientas tecnológicas externas a la universidad

Trabajó organizadamente en sus tareas

Utilizó provechosamente las herramientas tecnológicas de alguna plataforma de la universidad

$[0.00,0.95]$

2.40

$[1.24,3.55]$

1.20

$[0.38,2.02]$

1.65

$[0.69,2.61]$

1.50

$[0.58,2.41]$

$[1.35,3.74$

26.35

[24.89,31.69] [64.61,71.61]

$[23.02,29.67]$

59.13

8.23

[55.44,62.83]

$11.38 \quad 0.75$

2.10

[1.02,3.18]

[6.16,10.31]

43.11

$[8.98,13.78]$

3.74

$[39.39,46.84]$

46.56

34.58

[2.31,5.18]

$[31.00,38.16]$

10.93

[8.58,13.27]

56.89

11.08

[53.20,60.58]

57.04

[8.71,13.44]

$[53.33,60.74]$

19.91

56.89

[42.81,50.31]

2.84

[1.59,4.09]

[16.91,22.91]

[53.20,

5.54

23.20

45.66

59.13

[55.44,62.82]

[3.90,7.18]

$[20.23,26.18]$

$[42.26,49.06]$
29.19

[25.78,32.60]

$28.59 \quad 1.20$

25.20,31.99]

18.71

[15.79,21.64]

$15.27 \quad 10.33$

$2.71,17.83]$

Fase de reflexión

Reflexionó sobre el aprendizaje adquirido

Profundizó sus conocimientos en los temas tratados

Realizó investigaciones complementarias a los temas tratados

$\begin{array}{ccccc}2.10 & 20.81 & 64.22 & 11.53 & 1.35 \\ {[1.02,3.17]} & {[17.76,23.86]} & {[60.66,67.79]} & {[9.12,13.93]} & \\ 4.49 & 36.53 & 50.00 & 7.63 & 1.35 \\ {[2.93,6.05]} & {[32.91,40.14]} & {[46.26,53.74]} & {[5.64,9.63]} & \\ 10.48 & 41.77 & 39.67 & 6.74 & 1.35 \\ 8.17,12.78] & {[38.07,45.46]} & {[36.00,43.34]} & {[4.85,8.62]} & \end{array}$


Es sobresaliente el elevado porcentaje de estudiantes del GEP (93.71\%) que utiliza exitosamente las herramientas tecnológicas externas a la universidad, y la marcada intensidad con que alcanzan este logro (59.13\% en "Muy de acuerdo").

Los datos de la Tabla 4 muestran que la gran mayoría de la población estudiantil cumple con los plazos para entregar los trabajos (96.4\%) y realizan a tiempo las actividades extraclase (89.67\%). No obstante, aunque la mayoría considera que logra una adecuada dosificación del tiempo para realizar sus tareas existe un porcentaje significativo $(28,75 \%)$ de estudiantes que no lo logra.

Algunos resultados de la Tabla 4 se sintetizan en la Figura 4, en ella se pueden observar logros durante la última fase de la autorregulación del aprendizaje.

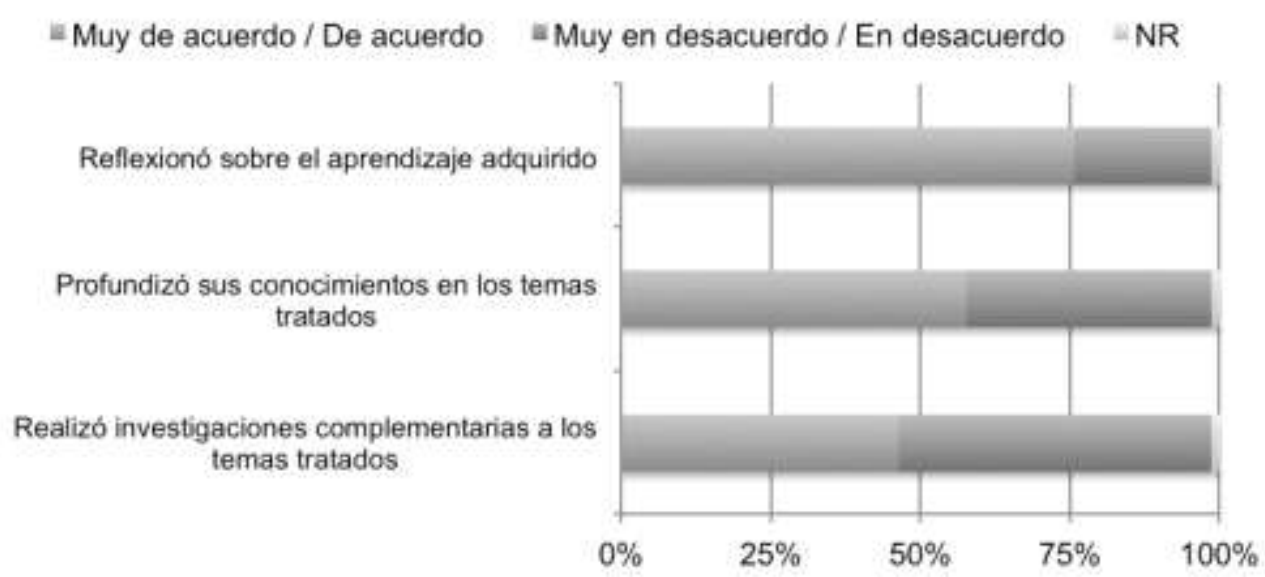

Fig. 4: Logros durante la fase de reflexión del proceso de autorregulación del aprendizaje en PLE

Como se muestra en la Figura 4, más de un 75\% de los estudiantes logran reflexionar sobre el aprendizaje adquirido. No obstante, durante la fase de reflexión, porcentajes significativos de estudiantes no realizan investigaciones complementarias a los temas tratados en la asignatura $(52.25 \%)$ o no profundizan los temas tratados $(41.02 \%)$.

\section{Relación entre los logros de los estudiantes}

La Tabla 5 muestra las correlaciones entre los logros que han alcanzado los estudiantes del GEP en la autorregulación del aprendizaje en sus PLE, los resultados más significativos (mayores o iguales a .30) se muestran en cursiva. Los datos de esta tabla muestran que la realización de investigaciones complementarias y la profundización de los temas tratados, ambos logros de la fase de actuación, están significativamente relacionados entre sí; además, ambos están positivamente correlacionadas con la reflexión del aprendizaje.

También, de la Tabla 5 se desprende que existe otro grupo de tres logros de la fase de actuación que están significativamente relacionados entre sí, estos son la adecuada dosificación del tiempo, el trabajo organizado y la realización de los trabajos extra-clase. Estos dos últimos logros también tienen una correlación fuerte con el cumplimiento de plazos para entregar los trabajos.

Si se tiene en cuenta que hay dos grupos de logros en los que se observan significativas correlaciones y se consideran las altas frecuencias que estos logros muestran en la Tabla 4, podría hablarse de la existencia de un subgrupo de estudiantes que alcanzan altos niveles de logros en investigación, profundización y reflexión, y un subgrupo de estudiantes que alcanzan altos niveles de logros en organización, dosificación del tiempo y cumplimiento de trabajos. La significativa relación entre la profundización de los conocimientos (del primer grupo de logros) y el cumplimiento de los plazos para entregar los trabajos (segundo grupo de logros) parece indicar que podría haber alguna relación entre estos dos grupos de estudiantes del GEP. Sin embargo, no hay ninguna otra correlación significativa entre estos dos grupos de logros.

\section{Efecto de las acciones sobre los logros en la autorregulación del aprendizaje en PLE}

Los resultados en la prueba de análisis de varianza Kruskal-Wallis de la Tabla 6 muestran el efecto de las acciones de los estudiantes GEP sobre los logros alcanzados por ellos, durante el proceso de autorregulación del aprendizaje en PLE. Estos resultados muestran que la acción de adaptar las herramientas de algún LMS de la universidad a las metas de aprendizaje tiene un efecto estadísticamente 
significativo sobre la realización de dos logros, uno de estos es la realización de investigaciones complementarias y el otro es la profundización de los conocimientos. Esto significa que más de un 10\% de la variación en dichos logros alcanzados por los estudiantes están explicadas por esta acción durante la autorregulación de los aprendizajes.

Tabla 5: Relación entre los logros en la autorregulación del aprendizaje en PLE, fases de actuación y reflexión. En la tabla, $N=1774$, (1) $p<0,01$, dos colas y (2) IC 95\%

Coeficiente de correlación de Kendall(1) e intervalos de confianza(2)

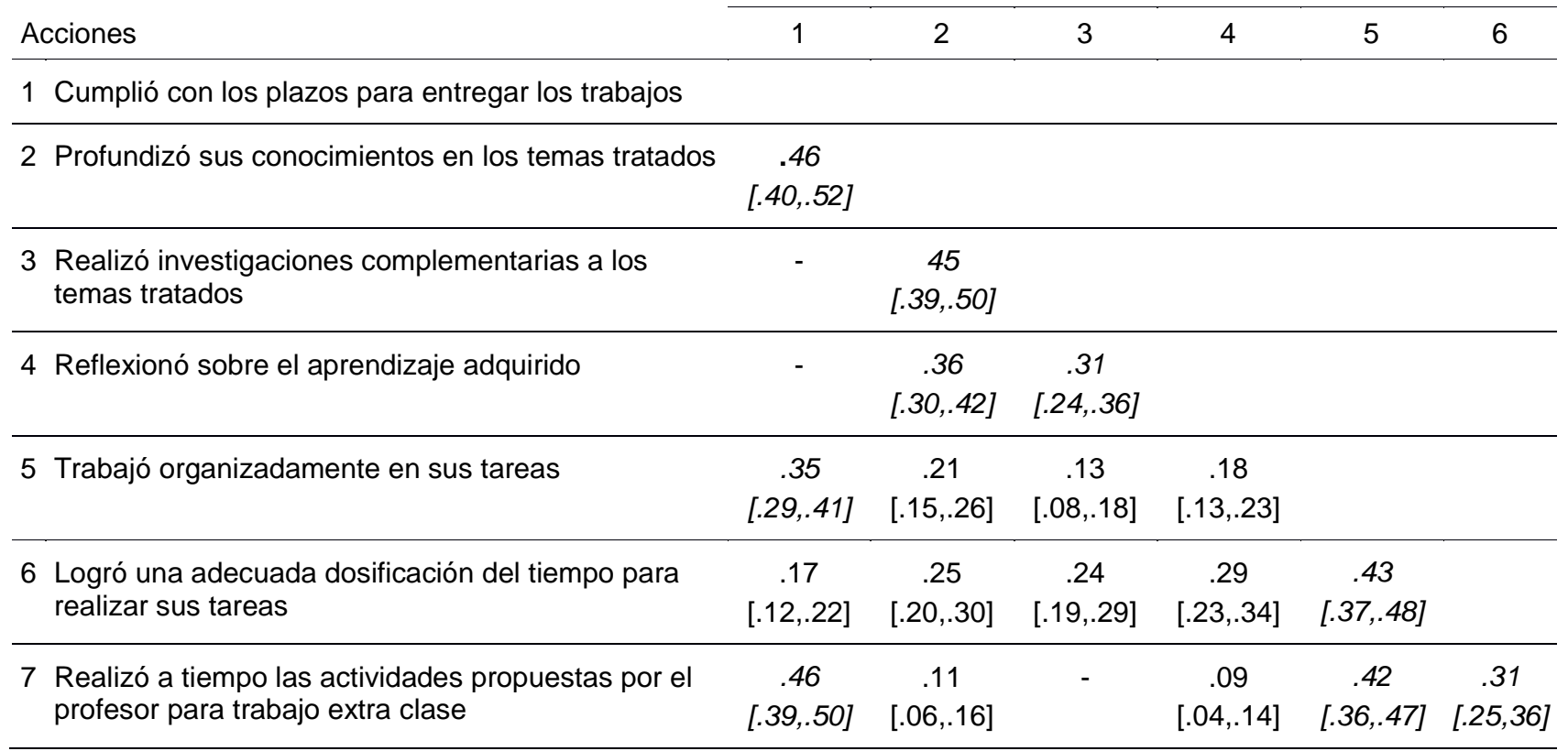

Por su parte, al observar la Tabla 6 se deduce que la acción de reflexionar sobre el papel en el propio aprendizaje explique en un 14\% que los estudiantes del GEP logren la reflexión del aprendizaje adquirido. También, de esto datos puede desprenderse que la acción de utilizar las herramientas externas a la universidad para intercambiar información con los compañeros de asignatura explica, en un $14 \%$, que los estudiantes logren utilizar exitosamente estas herramientas externas. Finalmente, la Tabla 6 indica que el uso del blog personal para profundizar ideas o conceptos de la asignatura influye positivamente, en casi un $10 \%$, sobre la realización de investigaciones complementarias en dicha asignatura.

Tabla 6: Efecto de las acciones sobre los logros durante la autorregulación del aprendizaje en PLE. En la tabla, $\mathrm{N}=1774, \mathrm{p}<0.01$, dos colas

Resultados en la prueba Kruskal-Wallis para análisis de varianza con tamaño del efecto e intervalos de confianza

\begin{tabular}{lll} 
& TE 95\% \\
\cline { 2 - 3 } Acciones & IC 95\% Logros
\end{tabular}

\begin{tabular}{lcc}
\hline $\begin{array}{l}\text { Utilizar herramientas externas a la universidad } \\
\text { para intercambiar información con personas } \\
\text { participantes en la asignatura }\end{array}$ & $\begin{array}{c}.38 \\
{[.29, .46]}\end{array}$ & $\begin{array}{l}\text { Utilizó exitosamente herramientas } \\
\text { tecnológicas externas a la } \\
\text { universidad }\end{array}$ \\
\hline $\begin{array}{l}\text { Reflexionar sobre el papel que tiene en el propio } \\
\text { aprendizaje }\end{array}$ & $\begin{array}{c}.37 \\
{[.27, .45]}\end{array}$ & $\begin{array}{l}\text { Reflexionó sobre el aprendizaje } \\
\text { adquirido }\end{array}$ \\
\hline $\begin{array}{l}\text { Utilizar un blog personal para profundizar ideas o } \\
\text { conceptos de la asignatura }\end{array}$ & $\begin{array}{c}.31 \\
{[.22, .39]}\end{array}$ & $\begin{array}{l}\text { Realizó investigaciones } \\
\text { complementarias a los temas } \\
\text { tratados }\end{array}$ \\
\hline $\begin{array}{l}\text { Adaptar las herramientas de alguna plataforma } \\
\text { LMS de la Universidad a las metas de } \\
\text { aprendizaje }\end{array}$ & $\begin{array}{c}.33, .41] \\
.32\end{array}$ & $\begin{array}{l}\text { Profundizó sus conocimientos en los } \\
\text { temas tratados }\end{array}$ \\
\hline
\end{tabular}




\section{ANÁLISIS DE RESULTADOS}

En la fase de actuación los estudiantes del GEP han incorporado algunas herramientas de LMS universitarios a sus PLE y las han adaptado a su aprendizaje. Durante esta fase los estudiantes prefieren las herramientas externas a la universidad para intercambiar información con sus compañeros de asignatura. Durante la fase de reflexión las herramientas digitales del PLE son importantes para que los estudiantes documenten y organicen la reflexión sobre su propio aprendizaje. Los profesores son un elemento sobresaliente en ambas fases del proceso de autorregulación del aprendizaje de los estudiantes. Es recomendable que los encargados de programas y los docentes participantes de los programas formativos tomen en cuenta que los estudiantes, como parte de sus acciones para autorregular el aprendizaje, solicitan el apoyo del profesor, valoran sus evaluaciones y muestran un pensamiento crítico ante sus estrategias de enseñanza.

La elevada correlación, durante la fase de actuación, entre intercambiar información de compañeros de asignatura con herramientas LMS universitarios y el hacerlo con herramientas externas a la universidad indica que estas herramientas no son sustitutas sino complementarias. También, durante la fase de actuación hay una significativa relación entre lograr adaptar herramientas LMS al PLE y el lograr adaptar estas herramientas a las metas de aprendizaje del estudiante. Esto podría indicar que una herramienta LMS es asumida en el PLE cuando el individuo logra adaptarla a las propias metas de aprendizaje. Por su parte, durante esta fase de la autorregulación del aprendizaje en PLE, las herramientas LMS universitarias y las externas a la universidad son complementarias para realizar la acción de intercambiar información con compañeros de asignatura. Estos hechos abren caminos para futuras investigaciones sobre la integración entre LMS y PLE.

Los datos demuestran que existe correlación entre la acción de utilizar herramientas digitales para representar ideas en formas diversas, realizada por los estudiantes en la fase de actuación, y dos acciones metacognitivas realizadas durante la fase de reflexión, correspondientes a reflexionar sobre el propio aprendizaje y valorar las sugerencias del profesor. En este punto se debe tomar en cuenta que la capacidad del estudiante para representar las ideas en formas diversas requiere de un adecuado dominio técnico sobre el uso de las herramientas físicas y virtuales del PLE. A partir de estos resultados es recomendable investigar, en futuros trabajos, si una mayor capacidad técnica del estudiante (que le permita presentar ideas con diversos formatos, utilizando diversas herramientas y en diversos entornos) puede favorecer que el estudiante cambie la estructura de sus ideas, las ordene y las relacione de otras maneras, y estudiar de qué manera esto lo ayuda en sus acciones reflexivas y metacognitivas.

Los estudiantes de la población consideran que son exitosos en las fases de actuación y de reflexión del proceso de autorregulación del aprendizaje en PLE. Los estudiantes perciben que administran bien su tiempo, cumplen con sus tareas, utilizan exitosamente las herramientas tecnológicas, gestionan bien la información y reflexionan sobre el aprendizaje adquirido. Sin embargo, hay cantidades significativas de estudiantes que no logra una adecuada dosificación del tiempo para realizar sus tareas, no logran realizar investigaciones complementarias o no profundizan los temas tratados. Es recomendable la realización de investigaciones más profundas para determinar en qué aspectos y por qué razones algunos estudiantes del GEP no alcanzan estos logros.

Durante la etapa de reflexión en PLE, logros como la realización de investigación complementaria y la profundización de los temas tratados están relacionada entre sí, y también, están relacionadas con el lograr realizar reflexión metacognitiva. Este hecho es importante para los encargados de programas formativos e investigadores, ya que cualquier acción didáctica que promueva algunos de estos logros, muy probablemente, servirá para fortalecer los otros dos. Gracias al análisis correlacional entre los logros de los estudiantes, se identifica un subgrupo de estudiantes que considera tener un alto nivel de logros en investigación complementaria, profundización de los temas y la reflexión meta cognitiva. También, se identifica un subgrupo de estudiantes con altos niveles de logro en organización, dosificación del tiempo y cumplimiento de tareas. Se recomienda investigar con mayor profundidad estos subgrupos de estudiantes.

Mediante al análisis de varianzas se ha logrado establecer la influencia de algunas acciones que realizan los estudiantes de la población para autorregular el aprendizaje en sus PLE, sobre logros que han alcanzado estos estudiantes en dicho proceso, durante las fases de actuación y reflexión. De esta manera se ha logrado determinar que la acción de adaptar las herramientas LMS a las metas de aprendizaje, durante la fase de actuación, influye significativamente sobre logros en la fase de reflexión, como la realización de investigaciones complementarias y la profundización de los conocimientos. Este resultados es coherente con la teoría, que señala la interconexión entre las distintas fases del proceso de autorregulación del aprendizaje; al tiempo que hace recomendable la adaptación de las herramientas LMS a las metas de aprendizaje de los estudiantes para lograr mayor profundidad temática y más investigación complementaria. 
No sorprende que la acción de reflexionar sobre el papel en el propio aprendizaje determine que los estudiantes logren la reflexión del aprendizaje adquirido, porque es una acción que corresponde directamente con el logro. Sin embargo, es más reseñable notar que el intercambio de información entre compañeros de asignatura, utilizando herramientas externas a la universidad, sea determinante para alcanzar una utilización exitosa de estas herramientas. La relación entre esta acción y este logro durante la fase de actuación no es tan obvia, es indicativa de la importancia que tienen estas herramientas en la comunicación entre compañeros de asignatura y del apoyo que se brindan entre sí para una exitosa utilización de estas herramientas.

Durante la fase de reflexión el uso del blog personal para profundizar ideas o conceptos de la asignatura influye positivamente sobre la realización de investigaciones complementarias en dicha asignatura. Esto indica la benéfica influencia de la profundización de ideas y de conceptos, también señala el potencial de los blogs para el aprendizaje.

\section{CONCLUSIONES}

Del estudio realizado con los estudiantes del GEP, se obtienen algunas conclusiones relacionadas con las fases de actuación y de reflexión del proceso de autorregulación del aprendizaje en PLE. Estas conclusiones se presentan a continuación.

Las herramientas digitales son importantes para las acciones estudiantiles de comunicación, de representación de ideas y de metacognición durante ambas fases del proceso de autorregulación de los aprendizajes en PLE, e incluye tanto las herramientas LMS universitarias como las externas a la universidad.

También, el papel de los profesores es determinante para las acciones autorregulatorias del aprendizaje, porque los estudiantes solicitan y valoran su apoyo; además, muestran un pensamiento crítico ante sus estrategias de enseñanza.

La población del estudio es exitosa en ambas fases del proceso de autorregulación del aprendizaje. Alcanzan logros significativos en el uso de herramientas digitales, en el cumplimiento de tareas y en la gestión de la información. Sin embargo, hay logros estudiantiles que deben reforzarse, como la dosificación del tiempo, la realización de investigación complementarias y la profundización sobre los temas de la asignatura.

En todos los programas pueden haber subgrupos de estudiantes que destacan en ciertos logros. En el caso del GEP un subgrupo de estudiantes destaca por sus logros en la organización, el cumplimiento de tareas y la administración del tiempo, el otro subgrupo destaca por la investigación, la profundización de los temas y la metacognición. Un mayor conocimiento sobre los subgrupos de estudiantes, según sus logros y sus características, puede orientar la búsqueda de mayores éxitos en la autorregulación del aprendizaje en PLE.

El uso adecuado de las herramientas digitales del PLE favorece la autorregulación del aprendizaje en ambas fases. Es recomendable incentivar en los estudiantes el uso educativo de las herramientas digitales externas a la universidad, así como ayudarles a adaptar las herramientas LMS universitarias a sus procesos de autorregulación del aprendizaje.

\section{REFERENCIAS}

Archee, R., Reflections on Personal Learning Environments: Theory and Practice, doi:10.1016/j.sbspro.2012.09.520, Procedia - Social and Behavioral Sciences, 55, 419-428 (2012).

Attwell, G., Personal learning environments-the future of elearning?, Elearning Papers, 2(1), 1-7 (2007a).

Attwell, G., E-portfolios - the DNA of the personal learning environment?, Journal of e-Learning and Knowledge Society, 3(2), 39-61 (2007b).

Azevedo, R., Understanding the complex nature of self-regulatory processes in learning with computerbased learning environments: An introduction, Metacognition Learning, 2, 57-65 (2007).

Bandura, A., Self-efficacy: The exercise of control, 1를 edición, 1-604, W.H. Freeman, New York, USA (1997).

Chatti, M. A. y otros tres autores, Toward a Personal Learning Environment Framework, International Journal of Virtual and Personal Learning Environments, 1(4), 66-85 (2010).

Cohen, J., Quantitative methods in psychology: A power primer, Psychological Bulletin, 112(1), 155-159 (1992). 
Dabbagh, N. y A. Kitsantas, Personal Learning Environments, social media, and self-regulated learning: A natural formula for connecting formal and informal learning, doi:10.1016/j.iheduc.2011.06.002, The Internet and Higher Education, 15(1), 3-8 (2012).

Drexler, W., The networked student model for construction of personal learning environments: Balancing teacher control and student autonomy, Australasian Journal of Educational Technology, 26(3), 369-385 (2010).

Ebner, M. y B. Taraghi, Personal learning environment for higher education: A first prototype, Actas del Congreso Proceedings of world conference on educational multimedia, hypermedia and telecommunications, 1158-1166, Chesapeake VA, USA, 29 de junio al 2 de julio (2010).

Fredes, C. A., J. P. Hernández y D. A. Díaz, Potencial y Problemas de la Simulación en Ambientes Virtuales para el Aprendizaje, Formación Universitaria, 5(1), 45-56 (2012).

Godwin-Jones, R., Emerging technologies: Personal learning environments, Language Learning y Technology, 13(2), 3-9 (2009).

Hadwin, A. F. y otros tres autores, Innovative ways for using gStudy to orchestrate and research social aspects of self-regulated learning, Computers in Human Behavior, 26(5), 794-805 (2010).

Howell, D. C., Statistical methods for psychology, 8ª edición, 1-771, Cengage Learning, Belmont, USA (2010).

Jin, P. y R. Low, Enhancing motivation and self-regulated learning in multimedia environments, en Education policy, reform, and school innovations in the Asia-Pacific Region, 1를 edición, Association for Childhood Education International-Hong Kong and Macao, pp. 525-547, Hong Kong, China (2009).

Johnson, M. W. y O. Liber, The Personal Learning Environment and the human condition: from theory to teaching practice, doi:10.1080/10494820701772652, Interactive Learning Environments, 16(1), 3-15 (2008).

Johnson, M. W. y D. Sherlock, Beyond the Personal Learning Environment: attachment and control in the classroom of the future, doi:10.1080/10494820.2012.745434, Interactive Learning Environments, 22(2), 146164 (2014).

Kruskal, W. H. y W. A. Wallis, Use of ranks in one- criterion variance analysis, Journal of the American Statistical Association, 47, 583-621 (1952).

Lechuga, M. y otros tres autores, Utilización de Entornos Virtuales Educativos y Recursos Educativos Abiertos (OpenCourseWare) en cursos de Ingeniería Química de la Universidad de Granada, España, Formación Universitaria, 7(4), 3-14 (2014).

Leech, N., K. Barrett y G. Morgan, IBM SPSS for intemediate statistics: Use and interpretation, 4ª edición, 1293, Taylor and Francis Group, New York, USA (2011).

Liber, O. y M. Johnson, Personal Learning Environments, doi:10.1080/10494820701772645, Interactive Learning Environments, 16(1), 1-2 (2008).

Pajares, F., Gender and perceived self-efficacy in self-regulated learning, Theory into Practice, 41, 116-225 (2002).

Pajares, F., Motivational role of self-efficacy beliefs in self- regulated learning, en Motivation and selfregulated learning: Theory, research, and applications, $1^{\text {a }}$ edición, Taylor and Francis Group, pp 111-140, New York, USA (2007).

Pintrich, P. R., A conceptual framework for assessing motivation and self-regulated learning in college students, Educational Psychology Review, 16(4), 385-407 (2004).

Pintrich, P. R. y otros tres autores, Predictive validity and reliability of the Motivated Strategies for Learning Questionnaire (MSLQ), Educational and Psychological Measurement, 53, 801-813 (1993).

Schunk, D. H. y B. J. Zimmerman, (Eds.)., Self-regulation of learning and performance: Issues and educational applications, $1^{\underline{a}}$ edición, 1-329, Lawrence Erlbaum Associates, Hillsdale NJ, Inglaterra (1994).

Schunk, D. H. y B. J. Zimmerman, Self-regulation and learning, en Handbook of psychology: Educational psichology, $1^{a}$ edición, Jhon Wiley y Sons.Inc, Vol. 7, pp 59-78, Hoboken NJ, USA (2003). 
Strahan, R. F., Assessing magnitude of effect from rank- order correlation coeffients, Educational and Psychological Measurement, 42, 763-765 (1982).

Tovar, L. C., J. A. Bohórquez y P. Puello, Propuesta Metodológica para la Construcción de Objetos Virtuales de Aprendizaje basados en Realidad Aumentada, España, Formación Universitaria, 7(4), 11-20 (2014).

Usher, E. L. y F. Pajares, Self-efficacy for self-regulated learning: A validation study, Educational and Psychological Measurement, 68, 443-463 (2008).

Väljataga, T. у M. Laanpere, Learner control and personal learning environment: A challenge for instructional design, doi:10.1080/10494820.2010.500546, Interactive Learning Environments, 18(3), 277-291 (2010).

Wilson, S., Patterns of Personal Learning Environments, doi:10.1080/10494820701772660, Interactive Learning Environments, 16(1), 17-34 (2008).

Wilson, S. y otros cinco autores, Personal learning environments: Challenging the dominant design of educational systems, Journal of E-Learning and Knowledge Society, 2(3), 1-11 (2007).

Zimmerman, B. J., Attaining self-regulation: A social cognitive perspective, en Handbook of Self-regulation, 1를 edición, Elsevier Academic Press, pp13-39, San Diego CA, USA (2000)

Zimmerman, B. J., Self-regulated learning and academic achievement: An overview, Educational Psychologist, 25, 3-17 (1990).

Zimmerman, B. J., Theories of self-regulated learning and academic achievement: An overview and analysis, en Self-regulated learning and academic achievement: Theoretical perspectives, $2^{\underline{a}}$ edición, Lawrence Erlbaum Associates, pp 1-37, Mahwah NJ, USA (2001).

Zimmerman, B. J., Investigating self-regulation and motivation: Historical background, methodological development, and future prospects, American Educational Research Journal, 45(1), 166-183 (2008).

Zimmerman, B. J. y T. J. Cleary, Adolescents' development of personal agency, en Adolescence and education (Self-efficacy beliefs of adolescents), 1를 edición, Information Age Publishing, Vol. 5, pp 45-69, Greenwich CT, USA (2006).

Zimmerman, B. J. y T. J. Cleary, Motives to self-regulate learning, en Handbook of motivation at school, $1^{a}$ edición, Routledge, pp 247-264, New York NY, USA (2009).

Zimmerman, B. J. y D. H. Schunk (Eds.), Self-regulated learning and academic achievement: Theory, research and practice, 1ํㅡㄹ edición, 1-200, Springer-Verlag Publishing, New York NY, USA (1989). 